\title{
Chemoselective $\mathrm{C}-\mathrm{C} \sigma$-Bond Activation of the Most Stable Ring in Biphenylene
}

\author{
Richard Y. Kong, Mark R. Crimmin* \\ [a] Department of Chemistry \\ Imperial College London \\ Molecular Science Research Hub \\ 80 Wood Lane, W12 0BZ \\ London, UK \\ E-mail: m.crimmin@imperial.ac.uk \\ Supporting information for this article is given via a link at the end of the document
}

\begin{abstract}
The chemoselective cleavage of a six-membered aromatic ring in biphenylene is reported using an aluminium(I) complex. This type of selectivity is unprecedented. In every example of transition metal mediated $\mathrm{C}-\mathrm{C} \sigma$-bond activation reported to date, the reaction occurs at the central four-membered ring of biphenylene. Insight into the origin of chemoselecitivty was obtained through a detailed mechanistic analysis (isolation of an intermediate, DFT studies, activation strain analysis). We conclude that the divergent reactivity can be attributed to differences in both the symmetry and radial extension of the frontier molecular orbitals of the aluminium(I) fragment compared to common transition metal fragments.
\end{abstract}

Reactions that break the strong $\mathrm{C}-\mathrm{C} \sigma$-bonds of hydrocarbons are finding increasing use in synthesis. ${ }^{[1,2]}$ When more than one type of $\mathrm{C}-\mathrm{C} \sigma$-bond is present in the substrate, chemoselectivity becomes a central consideration. In nearly all cases, substrate bias dictates which $\mathrm{C}-\mathrm{C} \sigma$-bond breaks. Structural features such strained ring systems prejudice the reagent toward specific sites and determine the chemoselectivity of the reaction. Examples where reagent control overrides substrate bias are essentially unknown.

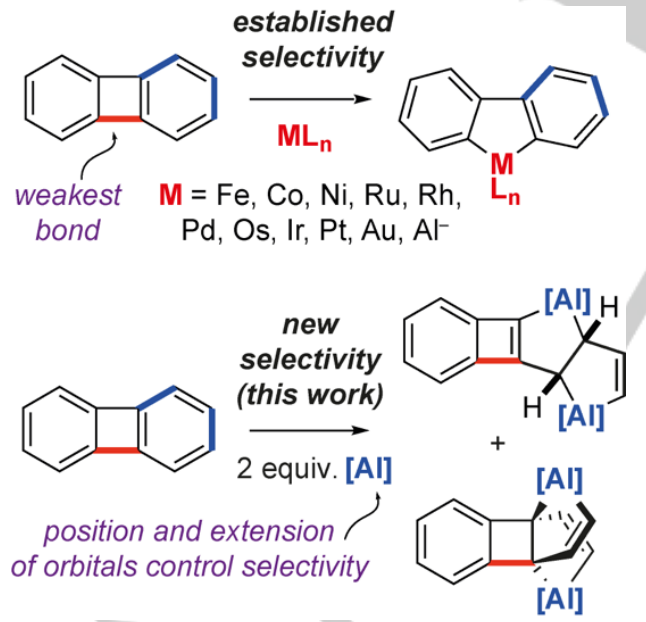

Scheme 1 Chemoselectivity in the $C-C \sigma$-bond activation of biphenylene.

Biphenylene has been the subject of detailed study in $\mathrm{C}-\mathrm{C} \sigma$ bond activation. The central $\mathrm{C}-\mathrm{C} \sigma$-bond of biphenylene has a low bond dissociation energy of $65.4 \mathrm{kcal} \mathrm{mol}^{-1}$. ${ }^{[3]}$ Both the antiaromatic character and strain of the four-membered ring contribute to the weakening of this $\mathrm{C}-\mathrm{C} \sigma$-bond. If biphenylene undergoes $\mathrm{C}-\mathrm{C} \sigma$-bond activation, this weakest link is always the site of reactivity. For example, the oxidative addition of a range of transition metal complexes to the central ring of biphenylene has been studied in great detail (Scheme 1a). ${ }^{[4-}$ ${ }^{8]}$ In cases where the substrate is asymmetric, directing groups can be used to define which $\mathrm{C}-\mathrm{C} \sigma$-bond of the central ring reacts. ${ }^{[9,10]}$ More recently, the reaction of the central $C-C \sigma$ bond of biphenylene with an anionic aluminium(I) nucleophile has been reported. ${ }^{[11]}$

The selectivity is unsurprising considering that alternative reactive sites of biphenylene require the dearomatisation of a benzene ring. The $\mathrm{C}-\mathrm{C}$ bond of benzene is strong and is enforced by a combination of $\sigma$ - and $\pi$-bonding. There are a number of transition metal[ ${ }^{[12-19]}$ and main group ${ }^{[20-24]}$ complexes that can effect the $\mathrm{C}-\mathrm{C} \sigma$-bond activation and dearomatisation of benzene units. Mechanistically, these systems show some common features: coordination of the metal complex to the arene $\pi$-manifold generates a strained metallocyclic intermediate from which $\mathrm{C}-\mathrm{C} \sigma$-bond activation evolves to form the observed products. None of these systems react with benzene units in the presence of more strained ring systems containing weaker $\mathrm{C}-\mathrm{C} \sigma$-bonds.

We recently reported the activation of $\mathrm{C}-\mathrm{C} \sigma$-bonds of alkylidene cyclopropanes using the aluminium(I) complex, 1 (Scheme 2). ${ }^{[25]}$ Here we demonstrate $\sigma-\mathrm{C}-\mathrm{C}$ bond activation of biphenylene using 1 . To the best of our knowledge, this is first example of $\mathrm{C}-\mathrm{C} \sigma$-bond activation with a main group reagent that leads to the direct installation of two metals into the fragmented carbon ring. Despite the substrate bias of biphenylene, the reaction is under reagent control and occurs with complete chemoselectivity for the strongest $\mathrm{C}-\mathrm{C} \sigma$-bonds of the six-membered arene ring. We provide a mechanistic rationale for this remarkable chemoselectivity and discuss its origin in the context of the divergent reactivity of transition metal and main group reagents.

The reaction of 2 equiv. of 1 with biphenylene at $100^{\circ} \mathrm{C}$ for 3 $h$ yields a mixture of 2 and 3 in a 1:0.7 ratio (Scheme 2). 2 and 3 are derived from the cleavage of the $C^{2}-C^{3}$ and $C^{4}-C^{5}$ bonds in biphenylene respectively. Both products incorporate metallocycles in which aluminium(III) centers are in 5membered rings. 2 crystallises readily from a concentrated toluene or THF solution of the reaction mixture at $-35^{\circ} \mathrm{C}$. 3 can be separated from 2 by extracting with THF and purified by recrystallising from toluene. Heating isolated samples of either $\mathbf{2}$ or $\mathbf{3}$ result in their decomposition and no interconversion is observed between these two compounds in either purified or crude mixtures. 


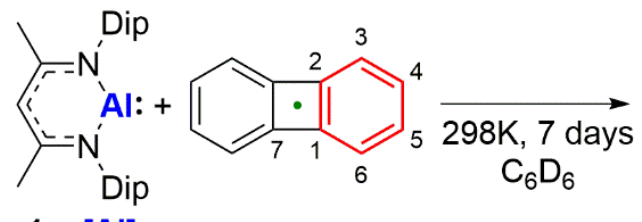

$\mathbf{1}=[\mathrm{Al}] \quad \operatorname{NICS}(0)=18.3$

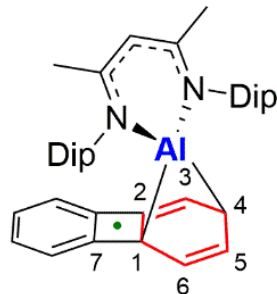

Isolated yield: $27 \%$

$\operatorname{NICS}(0)=5.3$

4

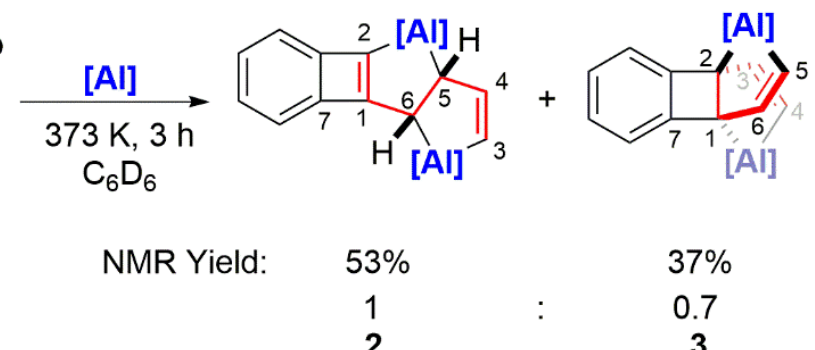

3

Scheme 2. $C-C \sigma$-Bond activation of biphenylene with 1 . Dip $=2,6$-di-isopropylphenyl.

Repeating the reaction of 1 with biphenylene at lower temperatures allowed the isolation of a reaction intermediate. After 7 days at $25^{\circ} \mathrm{C}$ addition of 1 to biphenylene yields 4 as a major product (Scheme 2). 4 is derived from a $(4+1)$ cycloaddition of 1 to the $\mathrm{C}^{1}$ and $\mathrm{C}^{4}$ positions of an arene ring of biphenylene. Based on cross-over experiments, the formation of $\mathbf{4}$ is, at least, partially reversible (See Supporting Information for further details). The formation of $\mathbf{4}$ can be explained by a pericyclic reaction, specifically a $\left[\pi_{\mathrm{s}} 4_{n} 2_{s}\right]$ cycloaddition. ${ }^{[26-29]}$ Formation of $\mathbf{4}$ generates a highly strained and dearomatized hydrocarbon framework that is primed for further reactivity. Addition of a further equiv. of $\mathbf{1}$ to 4 and heating to $100^{\circ} \mathrm{C}$ generates the 2 and 3 with similar yields and selectivity to that observed directly from 1 and biphenylene.

a)

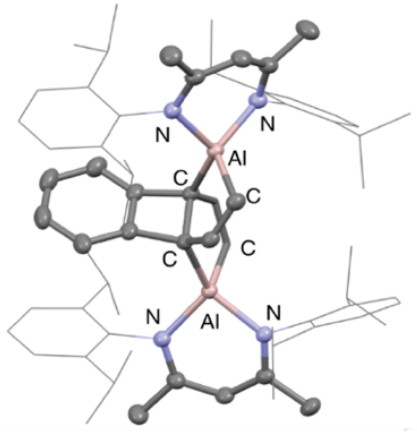

b)

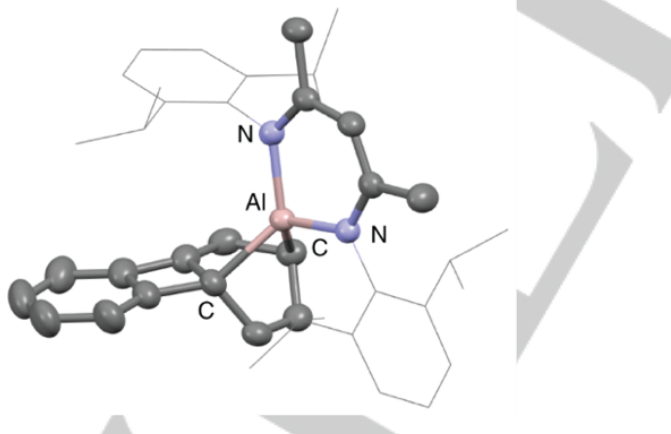

Figure 1. The structure of (a) 3 and (b) 4 by single crystal X-ray diffraction.

All products have been characterised by multinuclear NMR spectroscopy and single-crystal X-ray diffraction. The connectivity of $\mathbf{2}$ is evident in the solid-state structure however the ring-opened biphenylene unit is disordered over four sites (See Supporting Information for further discussion). Regardless, the ${ }^{1} \mathrm{H}$ NMR spectrum is consistent with the structural assignment. Characteristic alkene signals for the protons attached to $\mathrm{C}^{3}$ and $\mathrm{C}^{4}$ resonate at $\delta_{\mathrm{H}}=5.32$ and 7.44 ppm respectively. Compound 2 contains two contiguous stereocentres at the $\mathrm{C}^{5}$ and $\mathrm{C}^{6}$ position. The relative stereochemistry is determined by the cis-fused ring junction and has been confirmed by 1D NOE experiments. The crystal structure of 3 clearly shows the rupture of the aromatic ring. The aluminium centres of 3 are related by $C_{2}$-rotation around the perpendicular bisector of the $\mathrm{C}^{1}-\mathrm{C}^{2}$ bond. This compound again contains two contiguous stereocentres, now at the $\mathrm{C}^{1}$ and $\mathrm{C}^{2}$ positions, which impose a trans-fused ring junction and $\mathrm{C}_{2}$-symmetry (Figure 1 ).

DFT calculations were used to explore the mechanism of CC $\sigma$-bond activation (Figure 2). ${ }^{[30,31]}$ The $(4+1)$ cycloaddition of 1 to biphenylene was found to proceed via a single transition-state TS-1 $\left(\Delta \mathrm{G}^{\ddagger}{ }_{298 \mathrm{~K}}=28.5 \mathrm{kcal} \mathrm{mol}^{-1}\right)$ and forms 4 directly. Disruption of the antiaromaticity of the central ring of biphenylene likely determines the selectivity of the $(4+1)$ addition. NICS calculations show that this ring has less antiaromatic character in $4(\mathrm{NICS}(0)=5.3)$ than in biphenylene $(\operatorname{NICS}(0)=18.3)$ (Scheme 2). The $(4+1)$ cycloaddition of 1 to the $\mathrm{C}^{3}$ and $\mathrm{C}^{6}$ positions of biphenylene was also considered computationally. Both the transition state and product were found to be higher in energy than the pathway toward the formation of $\mathbf{4}$ (Supporting Information, Figure S5). Intermediate $\mathbf{4}$ has two chemically distinct alkene systems. 1 has previously been shown to perform $(2+1)$ cycloadditions with alkenes. ${ }^{[24,32-34]}$ Addition of 1 to the more sterically hindered alkene $\left(C^{2}=C^{3}\right)$ of 4 was found to proceed via a low energy transition state TS-2 to form Int-1. ${ }^{[25,34]}$ Int-1 is the product of an overall $4 \mathrm{e}^{-}$reduction of the arene ring of biphenylene with two equiv. of 1 . NBO analysis of TS-1 and TS-2 show that the nucleophilicity of $\mathbf{1}$ is a key driver towards the installation of the two aluminium centres (see supporting information for further detail). Int-1 incorporates two aluminium centres at the bridgehead positions of a norbornane $\left(\mathrm{Al}^{\mathrm{A}}\right)$ and norcarane $\left(\mathrm{Al}^{\mathrm{B}}\right)$ skeleton on opposite faces of the reduced arene ring.

Subsequent isomerisation of Int-1 to Int-2 occurs via transition state TS-3. This reaction involves a concerted rearrangement of the ring framework of Int-1 with two four-membered metallocycles forming from one three-membered and one fivemembered metallocycle. The relief of strain from the ringexpansion of the aluminocyclopropane is the likely thermodynamic driving force of this reaction. From Int-2 C-C $\sigma$-bond activation can occur by two different pathways. These pathways both involve a [1,3]-sigmatropic shift and a step that breaks the $\mathrm{C}-\mathrm{C} \sigma$-bond, however the order of these processes differs between the two. $\mathrm{C}^{2}-\mathrm{C}^{3} \sigma$-bond activation is possible directly from Int-2 via TS-4 to form Int-3. TS-4 is an early transition state in which a key bond cleavage step triggers a series of bond migrations to form Int-3. A subsequent [1,3]sigmatropic shift of $\left[\mathrm{Al}^{\mathrm{A}}\right]$ along the $\mathrm{C}^{3}-\mathrm{-}^{5}$ allyl system via TS5 results in formation of the observed product 2. 


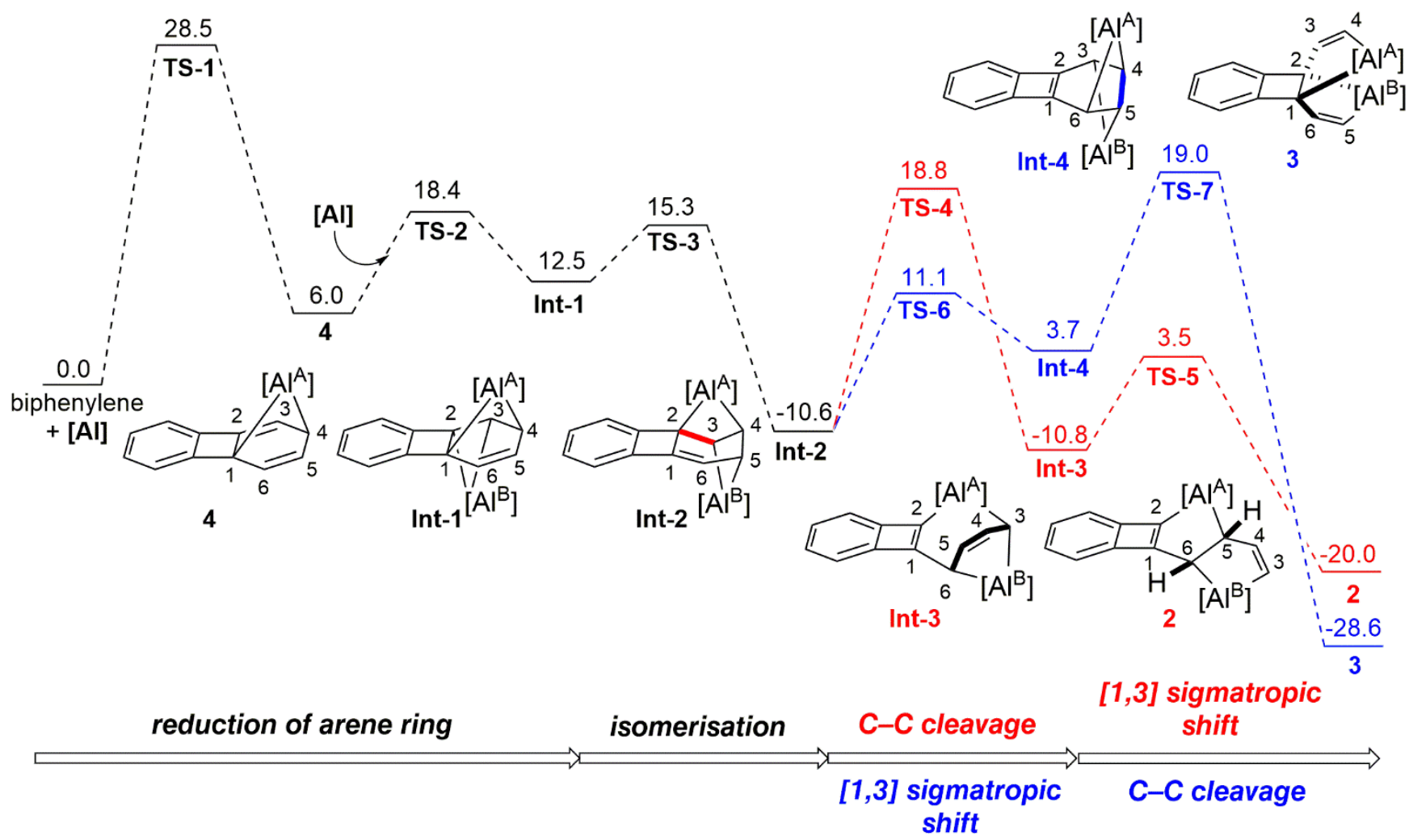

Figure 2. Calculated reaction pathway for the formation of $\mathbf{2}$ and $\mathbf{3}$ from $\mathbf{1}$ and biphenylene. Energies in $\mathrm{kcal} \mathrm{mol}^{-1}$. All calculations performed at the $\mathrm{M06I} / 6-31 \mathrm{G}^{* *}$ $(\mathrm{C}, \mathrm{H}, \mathrm{N}) / \mathrm{SDDAll}(\mathrm{Al})$ level of theory with single point dispersion (D3) and solvent (benzene) corrections.

Alternatively, $\left[\mathrm{Al}^{\mathrm{A}}\right]$ can perform a $[1,3]$-sigmatropic shift along the $\mathrm{C}^{2}--\mathrm{C}^{6}$ allyl system in Int-2 via TS-6 to form Int-4. The aluminium centres in Int- 4 are related by $C_{2}$-rotation around the perpendicular bisector of the $\mathrm{C}^{4}-\mathrm{C}^{5}$ bond and this symmetry is preserved into the product. Activation of the $\mathrm{C}^{4}$ $\mathrm{C}^{5} \sigma$-bond from Int-4 occurs via TS-7 and results in formation of 3.

The formation of both 2 and 3 is calculated to be highly exergonic and is consistent with these reactions being nonreversible. The $\mathrm{C}-\mathrm{C} \sigma$-bond activation steps have the highest activation barriers in the formation of $2\left(\Delta \mathrm{G}^{ \pm}{ }_{298 \mathrm{~K}}=29.4 \mathrm{kcal}\right.$ $\left.\mathrm{mol}^{-1}\right)$ and $3\left(\Delta \mathrm{G}^{ \pm} 298 \mathrm{~K}=29.6 \mathrm{kcal} \mathrm{mol}^{-1}\right)$ from 1 and biphenylene. ${ }^{[35]}$ In both cases, C-C $\sigma$-bond activation originates from a highly strained metallocyclic intermediate in which aluminium centers occupy positions in two separate metallocyclobutane rings. C-C cleavage via TS-4 and TS-7 relieves the strained 4-membered rings in Int-2 and Int-4 respectively. These steps occur without any significant change in the NPA charge at the aluminium(III) centres. The computationally predicted selectivity for the formation of $\mathbf{2}$ in preference to $3\left(\Delta \Delta \mathrm{G}^{\ddagger}{ }_{298 \mathrm{~K}}=0.2 \mathrm{kcal} \mathrm{mol}^{-1}\right)$ is consistent with experiment. The small $\left(\Delta \Delta \mathrm{G}^{\ddagger} \sim 1 \mathrm{kcal} \mathrm{mol}^{-1}\right)$ difference in activation energies between $\mathrm{C}-\mathrm{C} \sigma$-bond activation steps (TS4 and TS-7) and the (4+1) cycloaddition step (TS-1) is reflected experimentally: formation of $\mathbf{2}$ and $\mathbf{3}$ is observed during the synthesis of 4 .

The oxidative addition of 1 to the central $C^{1}-C^{7} \sigma$-bond of biphenylene was also explored computationally. While the overall reaction is highly exergonic $\left(\Delta \mathrm{G}_{298 \mathrm{~K}}=-64.9 \mathrm{kcal} \mathrm{mol}\right.$ $\left.{ }^{1}\right)$, the transition state is high in energy (TS-8, $\Delta \mathrm{G}^{\ddagger}{ }_{298 \mathrm{~K}}=42.0$ kcal mol${ }^{-1}$ ) and unlikely to be accessible under the reaction conditions (Supporting Information, Figure S4).

A deeper consideration of the key transition states can be used to rationalise the remarkable chemoselectivity of this system. Transition states for oxidative addition are typically destabilised by steric repulsion due to the requirement for a close - and side-on - approach of the $\mathrm{C}-\mathrm{X}$ bond to the metal to achieve orbital overlap (e.g. $\mathrm{X}=\mathrm{C}, \mathrm{H}$, Halogen). The oxidative addition of biphenylene to transition metal complexes has been calculated to involve a side-on approach and the synchronous breaking of the $\mathrm{C}-\mathrm{C} \sigma$-bond and formation of both $\mathrm{M}-\mathrm{C}$ bonds along the reaction coordinate..$^{[36,37]}$

1 differs from common transition metal fragments in terms of both the symmetry and energy of the frontier molecular orbitals. The HOMO and the LUMO of 1 are orthogonal to one another and the $3 p$ orbitals which contribute to them have a smaller radial extension than the $4 d$ and $5 d$ orbitals of $2^{\text {nd }}$ and $3^{\text {rd }}$ row transition metals. In line with these differences, TS-8 is unusual and is not consistent with a symmetric approach of the $\mathrm{C}-\mathrm{C}$ bond to the metal. TS-8 has an asymmetric geometry and involves close approach of the metal to only one carbon atom of the breaking $\mathrm{C}-\mathrm{C} \sigma$-bond.

Activation strain analysis (ASA) was used to interrogate this phenomenon further. ${ }^{[38-40]}$ Similar methodology has been used to explore the factors controlling $\mathrm{C}-\mathrm{H}$ and $\mathrm{C}-\mathrm{C}$ bond oxidative addition at an alumanyl anion. ${ }^{[11]}$ The ASA profile derived from TS-8 was generated (Figure $3 a$ ). In this profile, $E_{\text {Int }}$ decreases modestly along the reaction coordinate and a high degree of distortion in biphenylene is required before a 
favourable interaction between the two fragments is observed. This is further supported by the complex curvature of the reaction coordinate as a function of the $\mathrm{Al}-\mathrm{C}$ bond distance. For comparison, the profile associated with TS-1, and the $(4+1)$ cycloaddition is provided. While both TS-1 and TS-8 proceed via an initial nucleophilic attack of 1 onto biphenylene, the ASA profile TS-1 shows a steady increase in $E_{\text {dist }}(B i p h)$ that is offset by a steady decrease in $E_{\text {int }}$ (Figure $3 b$ ). The data allow us to conclude that the oxidative addition of the weak $\mathrm{C}-$ $\mathrm{C} \sigma$-bond of biphenylene to 1 is unfavourable due to the high degree of biphenylene reorganisation that is required for effective orbital overlap.

a)

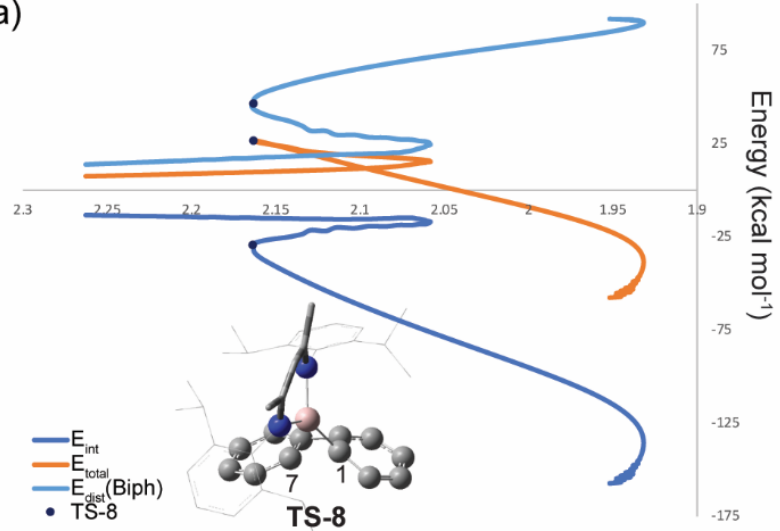

$\mathrm{Al}-\mathrm{C}^{7}$ bond distance $(\AA)$

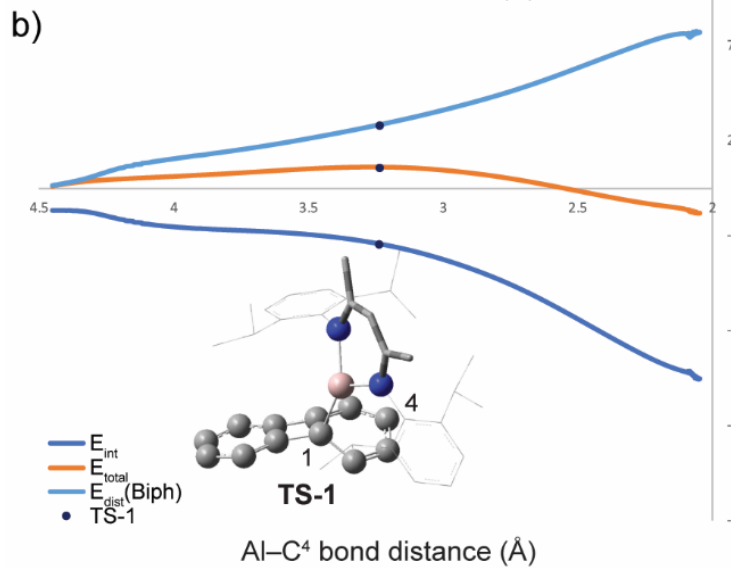

Figure 3. Activation strain analysis of the reaction profiles derived from TS-8 (a) and TS-1 (b).

In summary, we report a remarkable chemoselective $\mathrm{C}-\mathrm{C} \sigma-$ bond activation reaction involving the stable six-membered ring of biphenylene. This selectivity is unprecedented. Transition metal complexes react exclusively with the weakest $\mathrm{C}-\mathrm{C} \sigma$-bond of the four-membered ring of biphenylene, typically by an oxidative addition step. The divergent reactivity of 1 from transition-metal systems is a direct consequence of the orthogonality and limited radial extension of the frontier molecular orbitals of the aluminium reagent. This reagent cannot easily reach a stable transition state for oxidative addition of a $\sigma-C-C$ bond - due to the strain required to achieve orbital overlap - instead this it reacts preferentially with the flat and accesible $\pi$-system of the substrate opening up an alternative pathway for $\sigma-\mathrm{C}-\mathrm{C}$ bond activation.
X-ray crystallographic data 2-4 are available from the Cambridge Crystallographic Data Centre (CCDC 20237602023762) and as a .cif file, full details of the experiments and calculations are available as a .pdf. NMR spectra and computational coordinates are available at dx.doi.org/10.14469/hpc/7325.

\section{Acknowledgements}

We are grateful to the ERC (FluoroFix: 677367) for generous funding. We thank Imperial College London for the award of a President's Scholarship (RYK). Andrew J. P. White is thanked for helpful discussions on X-ray crystallography. ${ }^{[28]}$

Keywords: aluminium $\cdot \mathrm{C}-\mathrm{C}$ cleavage $\cdot$ arene activation $\cdot$ chemoselectivity $\cdot$ low-valent metals

[1] M. Murakami, N. Ishida, J. Am. Chem. Soc. 2016, 138, 13759-13769.

[2] R. Sarpong, B. Wang, M. A. Perea, Angew. Chem., Int. Ed. 2020, DO 10.1002/anie.201915657.

[3] W. D. Jones, in C-C Bond Activation (Ed.: G. Dong), Springer Berlin Heidelberg, Berlin, Heidelberg, 2013, pp. 1-31.

[4] J. J. Eisch, A. M. Piotrowski, K. I. Han, Carl. Kruger, Y. H. Tsay, Organometallics 1985, 4, 224-231.

[5] C. Perthuisot, W. D. Jones, J. Am. Chem. Soc. 1994, 116, 3647-3648.

[6] C. Perthuisot, B. L. Edelbach, D. L. Zubris, W. D. Jones, Organometallics 1997, 16, 2016-2023.

[7] A. B. Chaplin, R. Tonner, A. S. Weller, Organometallics 2010, 29, 2710 2714.

[8] H. Takano, T. Ito, K. S. Kanyiva, T. Shibata, Eur. J. Org. Chem. 2019, 2019 , 2871-2883.

[9] D. Frejka, J. Ulč, E. A. B. Kantchev, I. Císařová, M. Kotora, ACS Catal. 2018, 8, 10290-10299.

[10] H. Takano, T. Ito, K. S. Kanyiva, T. Shibata, Chem. Eur. J. 2018, 24, 15173 15177 .

[11] K. Koshino, R. Kinjo, J. Am. Chem. Soc. 2020, 142, 9057-9062.

[12] J. Browning, M. Green, A. Laguna, L. E. Smart, J. L. Spencer, F. G. A. Stone, J. Chem. Soc., Chem. Commun. 1975, 723-724.

[13] D. Ellis, D. McKay, S. A. Macgregor, G. M. Rosair, A. J. Welch, Angew Chem., Int. Ed. 2010, 49, 4943-4945.

[14] A. Sattler, G. Parkin, Nature 2010, 463, 523-526.

[15] P. D. Zeits, T. Fiedler, J. A. Gladysz, Chem. Commun. 2012, 48, 7925-7927. S. Hu, T. Shima, Z. Hou, Nature 2014, 512, 413-415.

G. Meola, H. Braband, D. Hernández-Valdés, C. Gotzmann, T. Fox, B. Spingler, R. Alberto, Inorg. Chem. 2017, 56, 6297-6301.

M. Jakoobi, N. Halcovitch, G. F. S. Whitehead, A. G. Sergeev, Angew. Chem., Int. Ed. 2017, 56, 3266-3269.

[19] M. Jakoobi, Y. Tian, R. Boulatov, A. G. Sergeev, J. Am. Chem. Soc. 2019, 141, 6048-6053.

[20] H. Suzuki, N. Tokitoh, R. Okazaki, J. Am. Chem. Soc. 1994, 116, 1157211573.

[21] D. Wendel, A. Porzelt, F. A. D. Herz, D. Sarkar, C. Jandl, S. Inoue, B. Rieger, J. Am. Chem. Soc. 2017, 139, 8134-8137.

[22] L. L. Liu, J. Zhou, L. L. Cao, R. Andrews, R. L. Falconer, C. A. Russell, D. W. Stephan, J. Am. Chem. Soc. 2018, 140, 147-150.

[23] L. Zhu, J. Zhang, C. Cui, Inorg. Chem. 2019, 58, 12007-12010.

[24] J. Hicks, P. Vasko, J. M. Goicoechea, S. Aldridge, J. Am. Chem. Soc. 2019, 141,11000-11003.

[25] R. Y. Kong, M. R. Crimmin, J. Am. Chem. Soc. 2020, 142, 11967-11971.

[26] S. Brand, H. Elsen, J. Langer, W. A. Donaubauer, F. Hampel, S. Harder, Angew. Chem., Int. Ed. 2018, 57, 14169-14173.

[27] R. J. Schwamm, M. D. Anker, M. Lein, M. P. Coles, Angew. Chem., Int. Ed. 2019, 58, 1489-1493.

[28] K. Sugita, R. Nakano, M. Yamashita, Chem. Eur. J. 2020, 26, 2174-2177.

[29] C. Bakewell, M. Garçon, R. Y. Kong, L. O'Hare, A. J. P. White, M. R. Crimmin, Inorg. Chem. 2020, 59, 4608-4616.

[30] X. Kang, G. Luo, L. Luo, S. Hu, Y. Luo, Z. Hou, J. Am. Chem. Soc. 2016, $138,11550-11559$.

[31] B. Zhu, W. Guan, L.-K. Yan, Z.-M. Su, J. Am. Chem. Soc. 2016, 138, 11069 11072 .

[32] H. Zhu, J. Chai, H. Fan, H. W. Roesky, C. He, V. Jancik, H.-G. Schmidt, M. Noltemeyer, W. A. Merrill, P. P. Power, Angew. Chem., Int. Ed. 2005, 44, 5090-5093.

[33] H. Zhu, R. B. Oswald, H. Fan, H. W. Roesky, Q. Ma, Z. Yang, H.-G. Schmidt, M. Noltemeyer, K. Starke, N. S. Hosmane, J. Am. Chem. Soc. 2006, $128,5100-5108$.

[34] C. Bakewell, A. J. P. White, M. R. Crimmin, Chem. Sci. 2019, 10, $2452-$ 2458. 
[35] The calculated energies reported at this level of theory suggest that Int-2 should be the isolable series. The trend of calculated energies reported is consistent across several different functionals (see SI for further details). Regardless, the formation of $\mathbf{4}$ is consistently observed experimentally with no other isolable products other than $\mathbf{2}$ and $\mathbf{3}$. The calculated energies will have some degree of error - especially in a step where two molecular fragments combine and accurate consideration of both entropy and dispersion contributions is required. The discrepancy between the isolable intermediate and computationally suggested intermediate likely arises from this error.

[36] Z. Gu, G. B. Boursalian, V. Gandon, R. Padilla, H. Shen, T. V. Timofeeva, P. Tongwa, K. P. C. Vollhardt, A. A. Yakovenko, Angew. Chem., Int. Ed. 2011 50, 9413-9417.

[37] H. Takano, N. Sugimura, K. S. Kanyiva, T. Shibata, ACS Omega 2017, 2 , $5228-5234$.

[38] This approach balances the energy penalty associated with distortion of the reagent fragments, $\left(E_{\text {dist }}\right)$, with the energy gain from their interaction $\left(E_{\text {int }}\right)$ along the reaction coordinate. As two bonds are forming over the course of both reactions, these values have been plotted against the lengths of the weaker $\mathrm{Al}-\mathrm{C}$ bond at the transition state (See Supporting Information for further discussion).

[39] F. M. Bickelhaupt, K. N. Houk, Angew. Chem., Int. Ed. 2017, 56, 1007010086 .

[40] I. Fernández, F. M. Bickelhaupt, Chem. Soc. Rev. 2014, 43, 4953-4967.

[41] J. J. Cabrera-Trujillo, I. Fernández, Chem. Eur. J. 2020, 26, 11806-11813. 
Entry for the Table of Contents $(5.5 \times 5.0 \mathrm{~cm})$

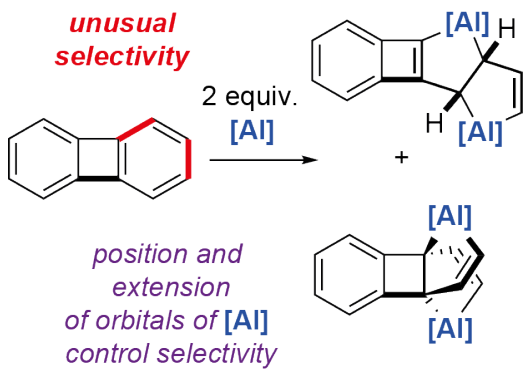

Al goes for Ar: The chemoselective cleavage of an arene ring in biphenylene is reported using an aluminium(I) complex. The reaction proceeds with complete integrity of the central four-membered ring despite this ring containing the weakest $\mathrm{C}-\mathrm{C} \sigma$-bond in the hydrocarbon scaffold. 